UCRL-ID-130358

\title{
Letter Report: Status on Code Maintenance (EQ3/6)
}

Thomas J. Wolery

April 24, 1995

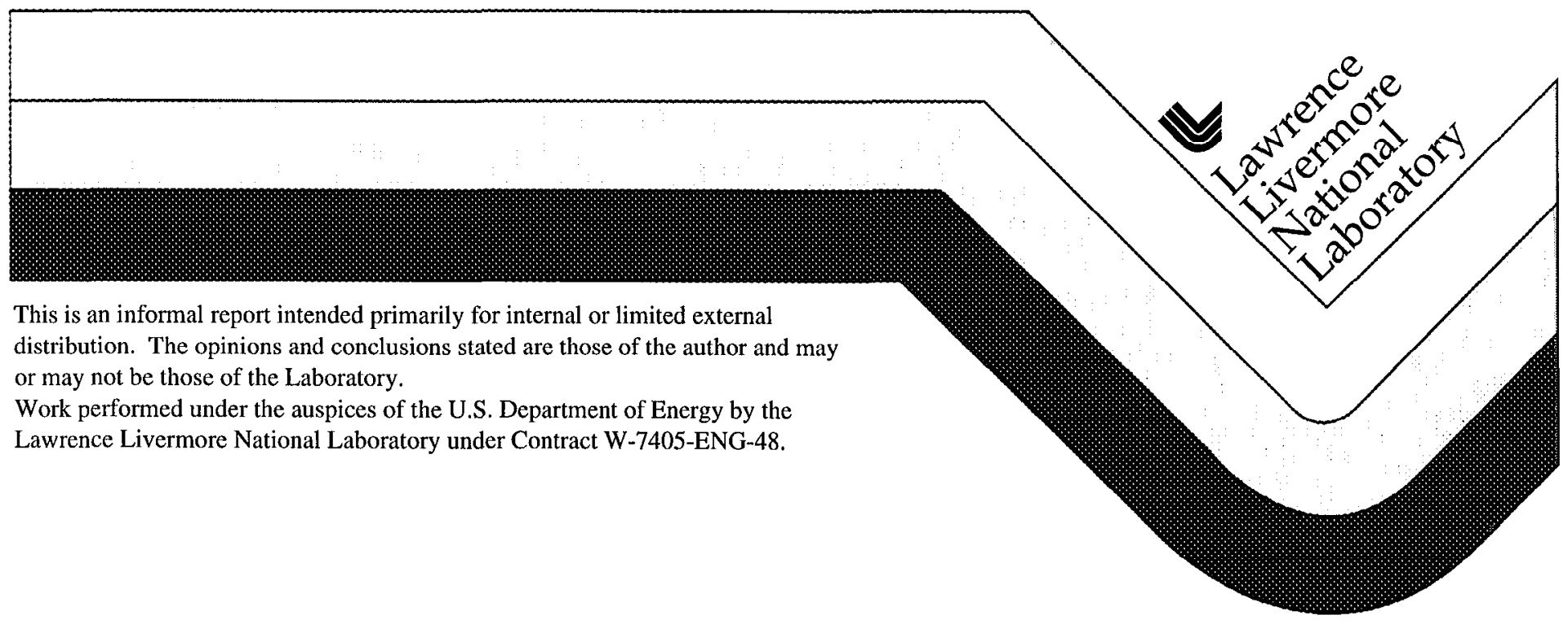




\section{DISCLAIMER}

This document was prepared as an account of work sponsored by an agency of the United States Government. Neither the United States Government nor the University of California nor any of their employees, makes any warranty, express or implied, or assumes any legal liability or responsibility for the accuracy, completeness, or usefulness of any information, apparatus, product, or process disclosed, or represents that its use would not infringe privately owned rights. Reference herein to any specific commercial product, process, or service by trade name, trademark, manufacturer, or otherwise, does not necessarily constitute or imply its endorsement, recommendation, or favoring by the United States Government or the University of California The views and opinions of authors expressed herein do not necessarily state or reflect those of the United States Government or the University of California and shall not be used for advertising or product endorsement purposes

This report has been reproduced directly from the best available copy

Available to DOE and DOE contractors from the Office of Scientific and Technical Information P O Box 62, Oak Ridge, TN 37831

Prices available from (423) 576-8401

Available to the public from the National Technical Information Service

US Department of Commerce 5285 Port Royal Rd Springfield, VA 22161 


\title{
LLNL Yucca Mountain Project \\ Near-Field Environment Characterization Technical Area
}

\author{
Letter Report: \\ Status on Code Maintenance (EQ3/6)
}

Thomas J. Wolery

April 24, 1995

University of California

$\leftrightarrows \begin{aligned} & \text { Lawrence Livermore } \\ & \text { National Laboratory }\end{aligned}$ 


\section{Status on Code Maintenance (EQ3/6), Letter Report, April 24, 1995}

\section{Status on Code Maintenance (EQ3/6)}

\section{Summary}

EQ3/6 is a software package for geochemical modeling of aqueous systems, such as water/rock or waste/water rock. It is being developed for a variety of applications in geochemical studies for the Yucca Mountain Site Characterization Project. Version 7.2a was the first version of this software to be certified for use in quality-affecting work (originally issued for use in non-quality-affecting work only on 12/28/93; certified on 8/17/94). In the past year, the Version 7 line software has been maintained while the new Version 8 line has been developed. In this period, sixtcen defect reports have been logged and resolved. Corrected software is being released as Version 7.2b. Defect reporting and resolution for the Version 7 line will continue until all released versions in this line are retired, perhaps six months to a year after Version 8.0 is released later this year. The Version 7 software is written in Fortran 77, technically speaking, but incorporates many aspects of older Fortran. The Version 8 software is written in a much more modern Fortran, technically somewhere between Fortran 77 and Fortran 90. Future code maintenance activities will include a more complete move to Fortran 90 , as well as continued maintaining of defect reporting and resolution.

\subsection{Introduction}

$\mathrm{EQ} 3 / 6$ and its usage have been extensively described in formal code documentation (Wolery, 1992ab; Daveler and Wolery, 1992; Wolery and Daveler, 1992). A more concise technical review is given by Wolery et al. (1990). Many other useful references may be found in these documents. EQ3/6 is being developed to model geochemical processes of relevance to the Yucca Mountain Site Characterization Project (YMSCP). The range of applications is broad, including studies pertaining to ambient groundwater geochemistry, waste form dissolution, and rock/water interactions in the altered zone. In some of this work the software is used to design or analyze experiments, in others, to predict processes that would occur in or about a repository. The software is also being applied in some natural analog studies. Various organizations participating in the YMSCP are using this software, including LLNL, LANL, LBL, PNL, Intera, and IT Corp., as well as number of cooperating foreign organizations including NAGRA BRGM, and W.S. Atkins Environment. At the direction of the DOE, the software has also been provided to the NWTRB and the CNWRA.

EQ3/6 is a package which contains various code elements. EQ3NR is a speciation-solubility code. It is used for example to compute the speciation of ions and solutes in groundwaters, and the saturation indices which define the saturation states of such waters with respect to various minerals. EQ6 is a reaction-path code. It allows one to compute simulations of interactions as among groundwaters and minerals. EQPT is a data file preprocessor. XCON3 and XCON6 are input file reformatters for EQ3NR and EQ6, respectively. EQLIB is a supporting library. In Version 8, some parts of this have been spun off into two new libraries, EQLIBU and EQLIBG. 


\section{Status on Code Maintenance (EQ3/6), Letter Report, April 24, 1995}

The first version of EQ3/6 to be certified for use in quality-affecting work was Version 7.2a. This was originally issued for use in non-quality-affecting work only on $12 / 28 / 93$. It was certified on $8 /$ $17 / 94$, after the completion of an independent $\mathrm{V} \& \mathrm{~V}$ (verification and validation) activity. This certification was restricted to use of the software on Sun SPARCSTATIONS, SGI workstations, and comparable UNIX platforms (exclusive of Crays) and on 386/486/Pentium type PCs.

Certification also excluded the use of the software for calculations involving solid solutions which are not ideal in either the molecular-mixing or the site-mixing sense (very little dala exists for the excluded models).

The Software Verification and Test Report (Kishi, 1995, in press; the draft of 7/12/94 has also been relained as a permanent record) described the independent $V \& V$ activity performed on Version 7.2a and the corresponding findings. A few mostly very minor errors were found, mostly sign errors in equations in the EQ3NR user's manual. One potentially significant error was found in the coding for non-ideal solid solutions which was written up as an exclusion (for this and other reasons) from the certification. It was decided that Version 7.2a should be certified, and that the errors found should be resolved along with any others reported in time in a Version 7.2b, which would be "born certified." Until the Version 7 software is retired, software quality assurance requirements mandate that a system be maintained for reporting and resolving reports of software defects. This is thus a minimal part of code maintenance.

At the same time, work had already been proceeding on the next development line, Version 8 . Whereas Version 7 was write in a Fortran 77 (ANSI, 1978) incorporating many older features and programming styles, Version 8 had been rewritten in a much more modern Fortran, technically between Fortran 77 and Fortran 90 (ANSI, 1991). The Fortran 90 features used were restricted to those available as "extensions" in existing Fortran 77 compilers. This rewriting had also been accompanied by a significant restructuring to allow the addition of new modeling capabilities. Three new major capabilities were added: thermodynamic pressure corrcctions, a gencric ionexchange capability, and the ability to model reaction-paths with redox disequilibrium. The Version 8 software is presently in beta testing, and is the current subject of another independent $\mathrm{V} \& \mathrm{~V}$ activity. Release of Version 8.0 (as certified software) is expected near the end of FY95. The Version 7 releases will be retried sometime thereafter. Version 8 will be maintained after the release of Version 8.0 until the releases in this development line are themselves retired.

\subsection{Code Maintenance}

Code maintenance is taken here to refer to three general kinds of activities. One is the continued adaptation required to keep up with changes in hardware, operating system software, programming language standards, and compilers. The second is the operation of a defect reporting and resolution system. The third is the construction of release packages of corrected code and their distribution to users. Other code activities (e.g., addition of new functional capabilities, or distribution of code with such new capabilities) are generally considered code development.

The move to Fortran 90 has been limited by the availability of suitable compilers. As noted above, some Fortran 90 features have been incorporated as "extensions" into Fortran 77 compilers. True 


\section{Status on Code Maintenance (EQ3/6), Letter Report, April 24, 1995}

Fortran 90 compilers are only now becoming available. We have recently obtained our first such compiler, the Lahey F90 compiler for PCs. We will obtain Fortran 90 compilers for our UNIX workstations sometime hopefully later in FY95. Over the next two years, we plan to move the Version 8 software increasingly into Fortran 90. This will allow a number of improvements to the software, including dynamic memory management and implementation of platform-independent coding, as for time and date functions. We will be engaging in both formal and informal staff training to assist in this work.

The defect reporting and resolution system used for EQ3/6 has been based on the use of a software-based system called CNGBOCHS (Daveler, Lundeen, and Johnson, 1993). This system is used by the Data Base Task, and is maintained by them. However, due to recent network management and other problems, CNGBOCHS has been almost impossible to use in the case of EQ3/6. Also, though it is possible for users to submit defect reports directly to CNGBOCHS using e-mail, no reports have been received in this manner over the past year. Several reports had however, been submitted directly to the Principal Investigator (PI) responsible for EQ3/6.

Because of the continued problems with CNGBOCHS and a backlog of reported defects, it was recently decided to drop CNGBOCHS as the primary system for defect reporting and resolution in favor of a system based on the use of a Software Defect Log maintained as an electronic document using FrameMaker. This document is similar to other existing electronic documents, including a Software Backup Log, a Software Development Log, and a Software Distribution Log. We may switch back to CNGBOCHS as the primary system for defect reporting and resolution once the local network has settled down.

All outstanding reports have been logged and resolved and are briefly described in the following sections, with three exceptions. These have been logged but not resolved. One (9504017) involves some calculations at a university involving solid solutions with local modification of the supporting thermodynamic data base, and involves a part of the software that was excluded from certification. Another (9504018) involves the development of EQ6 becoming stuck on small step sizes during kinetic runs. The third (9504019) involves a suspicious result in a brine-salt mineral solubility calculation reported by a German investigator. It may reflect a problem with the thermodynamic data base. These three reports have not yet been processed because of a staffing problem. They have been given low priority because they were not reported by YMP users. Only one (950418) appears to have any potential to affect YMP calculations. These reports will be addressed when additional staff have been obtained.

The defects described in the following section have been resolved in Version 7.2b. The release package for this version is presently being assembled and will shortly be distributed as an update to YMP users (distribution is expected to commence in May).

\subsection{Defect Reporting and Resolution}

All defect reporting and resolution in the past year is associated with the Version 7 line. The Version 8 software has been checked for the possible existence of the same defects found for Version 7. Sixteen defect reports were logged in the past year. Thirteen dealt with the software 


\section{Status on Code Maintenance (EQ3/6), Letter Report, April 24, 1995}

itself, and three purely with the documentation. The YMP users are being notified of these errors and of the availability of corrected code in Version 7.2b.

Of the thirteen reports dealing with the software itself, only two were considered serious. The first (9504005) was from the Software Verification and Test Report noted above. This is a potential indexing error in a part of the software dealing with non-ideal solid solutions. This part of the code was excluded from the certification of Version 7.2a for this and other reasons. This defect, an indexing error in the "do 26" loop in subroutine hpsat.f of the EQLIB library, was fixed. The other serious defect (9504010) is due to a compiler optimization error (in subroutine indat 1.f of the EQLIB library) and only affects the PC version. It may allow the fugacity of a gas which is supposed to be fixed in a reaction-path run to vary. It has also been fixed by altering the source code.

The remaining eleven reports dealing with defects in the software itself are either not defects at all, are harmless, cause problems only on unsupported platforms, or are only very minor in terms of impact. These are briefly described below.

A report (9504008) was made of a read format error when trying to run the software on a Sun SPARCSTATION running the Solaris operating system. This could not be verified, as the error could not be duplicated at LLNL and no further information could be obtained from the source of the report. The error appeared to have occurred in decoding a string containing time/date information provided by the system. It appeared that the string was not in the expected format. Although the error could not be duplicated, additional diagnostic coding was added to the software to trap such a situation.

A non-error (9504016) was a report that the coding for the Davies equation in subroutine gdavie.f of the EQLIB library contained an extra factor of 2 . In fact, this compensates for the fact that the equation is coded using the zsq 2 array, which contains one-half the charge squared, not the charge squared.

One harmless defect (9504001) concerned a missing "endif" in the RUNEQPT UNIX script. The script runs fine in all modes even with the defect. The defect was fixed anyway. Another harmless defect (9504002) concerned the use of an incorrect dimensioning parameter for the lomx array. The incorrect dimensioning parameter (nstpar) is larger than the correct one (nxtpar), so no harm resulted. This was also fixed anyway.

Another report (9504004) noted some instances of floating-point constants (e.g., "1.0")being used as arguments to the MIN and MAX. This can cause fatal compiler errors on some unsupported platforms if the type of the constants does not match the type of the other arguments (e.g., "1.0D0" is required instead of "1.0" if the other arguments are of type REAL*8. This problem was fixed by masking the type of the constants by using local variables matching the type of the other arguments). This is the normal practice in EQ3/6.

Another defect (9504006), harmless on supported platforms, is that in lambda.f of EQLIB, in the case of the binary solution third-order Maclaurin model, the variable "a" was nol unitized. Its proper value is zero. This defect was corrected. 


\section{Status on Code Maintenance (EQ3/6), Letter Report, April 24, 1995}

Another harmless error (9504007), relevant only to the PC version, is that the argument to the Lahey PC Fortran routine UNDFL in undflw.f of EQLIB should be type logical, not type integer. This reportedly does cause a problem with Lahey's new F90 compiler. This defect was also corrected.

A nuisance (9504009), but not really an error, is that EQ6 in kinetic mode jumps past the requested maximum model time to infinity. This is because the code does not actually adjust the step size so that a run ends at the requested maximum time; it merely stops once this time has been exceeded. This is made clear in the documentation (Wolery and Daveler, 1992).

A real but still nuisance-level error (9504003) is that in the gas fugacity tables written by the EQ6 code, the fugacity and log fugacity data were reversed. This error is obvious from an examination of the tables. The biggest problem is that the format for the log fugacity ill-suits the range of typical fugacity values. However, these can be obtained from the log fugacity values using a calculator. This defect was corrected by printing the affected variables in the order which matches the table header.

Floating-point underflow is a normal condition in EQ3/6. Nevertheless, on the Sun SPARCSTATION, an IEEE floating-point exception message warning of underflow is generated. This message is undesirable. In addition to possibly dulling the user into not recognizing more serious IEEE floating-point exception messages, warning for example of overflow, the message often appears in the middle of other code output. This defect (9504011) was fixed by writing a new subroutine written in C which accesses Sun's IEEE floating point interface and clears the underflow flag after a normal run.

Another problem (9504013), this one affecting the software only on the PC, is that the files associated with the "com" or composite data file all have a file name extension of "com". DOS and Windows generally recognize a file with such an extension to be a command file, which these files are not. No problem results when the software is run in a DOS window or under straight DOS. However, this does cause problems if attempting to run the software under the Windows Program Manager (see below). It is suggested that if this is done, the offending extension be changed to "cmp" or "cml".

.Of the three reports dealing only with the software documentation, one (9504014) was from the Software Test and Verification Report (cited above), and noted a number of errors in equations in the EQ3NR manual (Wolery, 1992b). These were mostly sign errors, but one missing equation was also noted. These errors will be documented in the published Software Test and Verification Report. They will be corrected in the EQ3NR manual itself when this is revised. The second reported error in code documentation (9504015) concerns an obvious typographical error in the EQPT manual (Daveler and Wolery, 1992). This will be corrected when this manual is revised. The error ("alpha 2 and alpha 2" for "alpha 1 and alpha 2" is obvious and does not adversely impact code usage; it is therefore harmless. The third defect report concerning documentation (9504012) was a complaint that no documentation was provided for running EQ3/6 on the PC under the Windows Programming Manager. We do not presently offer a means for doing this, but may do so in the future. We now recommend that the software be run in a DOS window or under straight DOS, and provide the necessary instructions for this. 


\section{Status on Code Maintenance (EQ3/6), Letter Report, April 24, 1995}

\subsection{Future Work}

We will continue to move the Version 8 line of code increasingly into Fortran 90 . We will formally document any defects reported in the beta testing phase, and continue defect reporting and resolution activities for this line until the last release in it has been retired. We will continue to maintain defect resolution and reporting activities for the Version 7 line until all released versions in this line have been retired. This is expected to occur within about six months to a year of the release of Version 8.0.

\section{References}

ANSI, 1978,Progamming Language FOR'TRAN: ANSI X3.9-1978, ISO 1539-1980 (E): American National Standards Institute, New York.

ANSI, 1991, Fortran 90: ANSI X3.198-1991, ISO/IEC 1539-1991 (E): American National Standards Institute, New York.

Daveler, S. A., Lundeen, S. R., and Johnson, J. W., 1993, CNGBOCHS: An Integrated IngresEmail-Interleaf System for Processing Change Requests Associated with GEMBOCHS, EQ3/6, and Other Research Groups: UCRL-ID-111991, Lawrence Livermore National Laboratory, Livermore, California.

Daveler, S. A., and Wolery, T. J., 1992, EQPT, A Data File Preprocessor for the EQ3/6 Software Package: User's Guide and Related Documentation (Version 7.0): UCRL-MA-1 10662-PTII, Lawrence Livermore National Laboratory, Livermore, California.

Kishi, T., 1995, EQ3/6 Software Test and Verification Report 7/94: UCRL-ID-in press, Lawrence Livermore National Laboratory, Livermore, California.

Wolery, T. J., Jackson, K. J., Bourcier, W.L., Bruton, C.J., Viani, B.E., Knauss, K.G., and Delany, J.M., 1990, The EQ3/6 software package for geochemical modeling: Current Status: In Melchior, D.C., and Bassett, R.L., eds., Chemical Modeling in Aqueous Systems II, American Chemical Society Symposium Series, 416, American Chemical Society, Washington, DC, p. 104-116.

Wolery, T. J., 1992a, EQ3/6, A Software Package for Geochemical Modeling of Aqueous Systems: Package Overview and Installation Guide (Version 7.0): UCRL-MA-110662-PTI, Lawrence Livermore National Laboratory, Livermore, California.

Wolery, T. J., 1992b, EQ3NR, A Computer Program for Geochemical Aqueous SpeciationSolubility Calculations: Theoretical Manual, User's Guide, and Related Documentation (Version 7.0): UCRL-MA-1 10662-PT-III, Lawrence Livermore National Laboratory, Livermore, California. 


\section{Status on Code Maintenance (EQ3/6), Letter Report, April 24, 1995}

Wolery, T. J., and Daveler, S. A., 1992, EQ6, A Computer Program for Reaction-Path Modeling of Aqueous Geochemical Systems: Theoretical Manual, User's Guide, and Related Documentation (Version 7.0): UCRL-MA-110662-PT-IV, Lawrence Livermore National Laboratory, Livermore, California. 


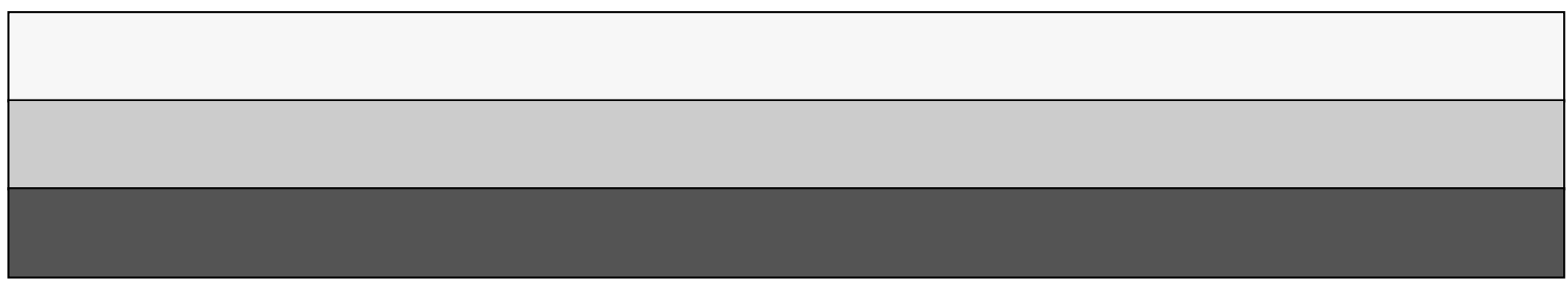

